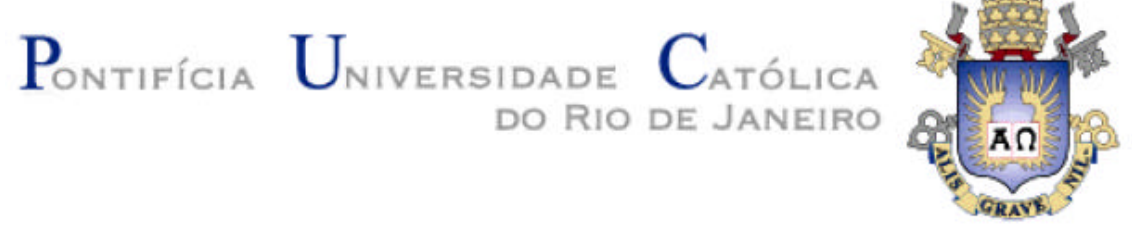

Augusto Mendonça Lessa

\title{
Avaliação de Valor em Uso de cargas ferríferas para Alto-Forno
}

\author{
Dissertação de Mestrado \\ Dissertação apresentada como requisito parcial para obtenção do grau de \\ Mestre em Engenharia Metalúrgica e de Materiais pelo Programa de Pós- \\ Graduação em Engenharia Metalúrgica do Departamento de Ciência dos Materiais \\ e Metalurgia da PUC-Rio
}

Orientador: Prof. José Carlos D’Abreu, DSc.

Rio de Janeiro

Agosto de 2009 
AUGUSTO MENDONÇA LESSA

Avaliação de Valor em Uso de cargas ferríferas para Alto-Forno

Dissertação apresentada como requisito parcial para obtenção do grau de

Mestre em Engenharia Metalúrgica e de Materiais pelo Programa de PósGraduação em Engenharia Metalúrgica do Departamento de Ciência dos Materiais

e Metalurgia da PUC-Rio. Aprovada pela Comissão Examinadora abaixo assinada.

Prof. José Carlos D`Abreu, DSc

Orientador

Departamento de Ciência dos Materiais e Metalurgia - PUC-Rio

Helio Marques Kohler, PhD

Consultor Independente da Samarco Mineração

Prof. Luiz Fernando Andrade de Castro, DSc

Universidade Federal de Minas Gerais - UFMG

Denilson Rodrigues Araújo, DSc

Samarco Mineração S.A

Prof. José Eugênio Leal

Coordenador Setorial de Pós-Graduação do Centro Técnico Científica da PUC-Rio

Rio de Janeiro, 14 de agosto de 2009. 
Todos os direitos reservados. É proibida a reprodução total ou parcial do trabalho sem autorização da universidade, do autor e do orientador

\section{Augusto Mendonça Lessa}

Graduou-se em Engenharia Metalúrgica e de Materiais na UFMG (Universidade Federal de Minas Gerais) em Dezembro de 2001.

Ficha Catalográfica

\section{Lessa, Augusto Mendonça}

Avaliação de valor em uso de cargas ferríferas para alto-forno / Augusto Mendonça Lessa ; orientador: José Carlos D’Abreu. - 2009.

124 f. : il. (color.) ; $30 \mathrm{~cm}$

Dissertação (Mestrado em Engenharia de Materiais)-Pontifícia Universidade Católica do Rio de Janeiro, Rio de Janeiro, 2009.

Inclui bibliografia

1. Engenharia de materiais - Teses. 2. Modelagem matemática. 3. Alto-Forno. 4. Pelotas. 5. Valor em uso. I. D’Abreu, José Carlos. II. Pontifícia Universidade Católica do Rio de Janeiro. Departamento de Engenharia de Materiais. III. Título. 


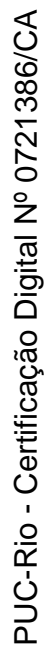

Dedico este trabalho à minha Déli. 


\section{Agradecimentos}

A execução de um de trabalho de mestrado é sempre uma atividade de grande esforço para o mestrando, porém acredito que seria impossível concluir esta atividade sem o auxílio e apoio de diversas pessoas e/ou instituições. Sou extremamente grato a estas pessoas e gostaria de deixar registrados meus sinceros agradecimentos.

À Samarco Mineração S.A por patrocinar e dar-me todo o subsídio para concluir esta atividade dentro do tempo esperado.

Aos diretores Roberto Carvalho e Ricardo Vescovi por acreditarem nesta linha de desenvolvimento e darem as diretrizes necessárias.

Ao Ricardo Melo, Helio Cardoso Pereira e em especial ao Haroldo Magalhães por terem passado os últimos anos gerenciando meu trabalho e incentivando a continuidade, mesmo nos momentos difíceis em que somos mais exigidos.

Ao meu pai, Francisco Dias Lessa, pelas incontáveis discussões e orientações sobre cada tema e abordagens que deveriam ser dadas. Além disso, e mais importante, pelo apoio moral e ético que você e dona Normanda sempre me passaram durante toda minha vida.

Ao Denilson, que me passou a demanda deste trabalho e sempre incentivou na evolução do conhecimento.

Ao professor Ronaldo Santos Sampaio, pelas várias orientações a respeito dos conceitos de valor em uso e de custos. Realmente o Sr. é especial, muito obrigado!

Ao Fernando Boechat pela amizade e grande ajuda na busca de referências bibliográficas. 
Aos amigos Francisco, Otaviano, Maurício Cota, Vinícius Fonseca, Renato e Leandro pelo apoio.

À Tatiana Silva e Virgínia pelos vários arranjos de viagens, outras atividades administrativas e pelos momentos de descontração.

Em especial ao Frederico Godinho, Guilherme Defendi, Fabiano Cristeli, pelo apoio e esclarecimentos a respeito do processo de Alto-Forno.

Ao Virgílio Gaggiato pelo auxílio na busca de dados de mercado.

Aos novos colegas Bruno e Plínio que estiverem sempre disponíveis para me ajudar nas atividades diárias.

À toda equipe do laboratório da Samarco.

À Nízia pela grande ajuda na revisão final do trabalho. 


\section{Resumo}

Lessa, Augusto Mendonça; D’Abreu, José Carlos. Avaliação de valor em uso de cargas ferríferas para Alto-Forno. Rio de Janeiro, 2009. 124p. Dissertação de Mestrado - Departamento de Engenharia de Materiais, Pontifícia Universidade Católica do Rio de Janeiro.

A indústria de minério de ferro tem importante papel na melhoria das condições de vida da população mundial por ser o principal insumo à produção de aço que, por sua vez, é o material mais utilizado e com as mais variadas aplicações no mundo. Neste contexto, é de suma importância o desenvolvimento tecnológico das empresas de mineração no âmbito de produzir seus minérios com mais qualidade, em maior escala e com menores custos para o benefício de toda a cadeia. Foi desenvolvido neste trabalho um modelo de medição do Valor em Uso de cargas metálicas no processo de Alto-Forno, que é a rota mais importante e responsável por $94 \%$ da produção mundial de ferro primário. Os resultados do modelo desenvolvido foram validados com dados reais de 23 Altos-Fornos de 7 países, os quais possuem práticas operacionais distintas. Este modelo apresentou um desvio médio de $4 \%$ entre os resultados obtidos e os dados de operação. O estudo de caso para cálculo de Valor em Uso foi realizado focando um dos 23 Altos-Fornos, operando com uma distribuição de carga metálica composta de sínter, pelota e lump, similar à média mundial. A proporção de pelotas na carga metálica foi variada de 17 a 50\%. Em um dos cenários avaliados, a adição de valor no processo siderúrgico, advinda desta variação, foi de 4,6\% na "margem de contribuição", e de 2,1\% na produtividade do Alto-Forno. Caso este ganho em valor fosse transferido ao preço do insumo pelota de minério de ferro, este poderia ser acrescido em 11\%. Os resultados obtidos evidenciam o quão importante é o desenvolvimento das ferramentas de análise de Valor em Uso para uma maior explicitação dos ganhos ou perdas proporcionados pelos diferentes tipos de minérios de ferro. Além disso, a análise de Valor em Uso demonstrou estar relacionada com as condições específicas do mercado e dos acordos comerciais 
estabelecidos e, sem dúvida, se constitui numa poderosa ferramenta para o desenvolvimento das indústrias de minério de ferro e aço.

\section{Palavras-chave}

Valor em Uso, Alto-Forno, Pelotas, Modelagem Matemática 


\begin{abstract}
Lessa, Augusto Mendonça; D’Abreu, José Carlos. Value in Use assesment of metalic burden to the Blast Furnace process. Rio de Janeiro, 2009. 124p. MSc Dissertation - Departamento de Engenharia de Materiais, Pontifícia Universidade Católica do Rio de Janeiro.

As steel is the most utilized and applied material in the world the iron ore industry has a very important role to society on improving human beings life conditions. It is of single importance the technological development of iron ore companies on producing higher quality materials, in larger scales and at lower costs creating value to the whole. It was developed an iron ore burden Value In Use assessment model for the Blast Furnace process which is responsible for producing $94 \%$ of all iron in the World. The outputs from the model were cross checked with real operational data of 23 Blast Furnaces with distinct practices, from 7 countries. The model exhibited an average deviation achieved was of $4 \%$ between the calculated outputs and the operational data set. For the case study one out of the 23 Blast Furnaces was chosen due its world average equivalent burden distribution in terms of sinter pellets and lump to perform an iron ore Value In Use assessment. Scenarios were built with pellet participation in the metallic burden varying from 17 to $50 \%$, achieving 4,6\% gains in the contribution margin and $2,1 \%$ incremental BF productivity in one of the scenarios. If all this gain is reverted to the pellet price, it could be increased by $11 \%$. The obtained results show how important the development of Value In Use analysis tools are as to revel true gains or losses from each iron ore type to the process. Further Value In Use analysis is an important tool to mining and steel industries and is always made against one reference case, relating it to a specific market and commercial conditions being applied to a single operation.
\end{abstract}

\title{
Keywords
}

Value In Use, Pellets, Blast Furnace, Mathematical Modeling 


\section{Sumário}

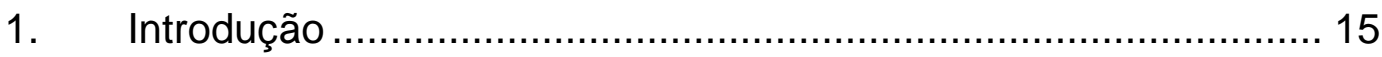

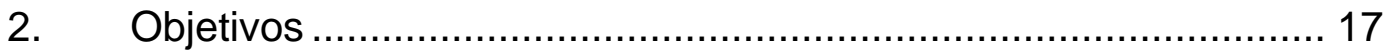

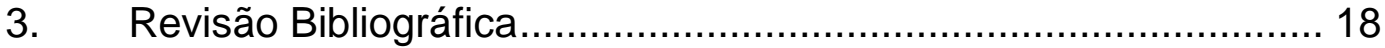

3.1. Evolução da produção de aço no mundo............................. 18

3.1.1. Histórico / Generalidades................................................... 20

3.2. Tecnologias relacionadas à obtenção de ferro gusa............. 26

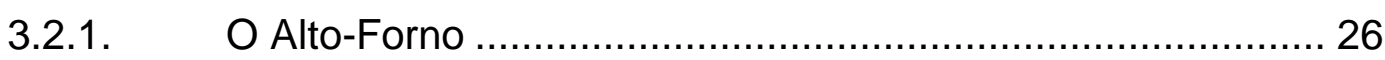

3.2.2. Equipamentos auxiliares do Alto-Forno ……..................... 27

3.3. Fundamentos do processo de redução de minério de ferro via

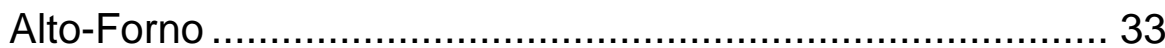

3.3.1. Parâmetros termodinâmicos relativos à redução de minério de ferro via Alto-Forno ................................................................ 40

3.3.2. Parâmetros cinéticos relativos à redução de minério de ferro via Alto-Forno....................................................................... 43

3.3.3. Principais matérias-primas empregadas na produção de ferro gusa via Alto-Forno ...................................................... 45

3.3.4 Critérios técnicos adotados para a seleção de cargas metálicas para o Alto-Forno ................................................5 50

3.3.4.1 Parâmetros ligados às características físicas de cargas metálicas utilizadas em Alto-Forno .................................... 51

3.3.4.2. Parâmetros ligados à qualidade química de cargas metálicas utilizadas em Alto-Forno ................................................. 53

3.3.4.3. Parâmetros ligados à qualidade metalúrgica de cargas metálicas utilizadas em Alto-Forno ...................................... 55

3.4. Importância do uso de pelotas de minério ferro na produção de ferro gusa via Alto-Forno.............................................. 57

3.5. Modelagem computacional e a Engenharia: conceitos básicos sobre modelagem computacional ....................................... 59

3.6. Modelagem computacional na produção de ferro gusa via

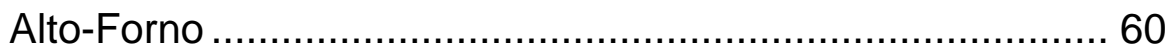

3.7. O modelo Termoquímico híbrido proposto............................ 62 
3.8. A abordagem de determinação do custo entregue de minérios de ferro baseado em dmtu ..................................................... 62

4. Desenvolvimento do modelo ...................................................... 72

4.1. Concepção............................................................... 72

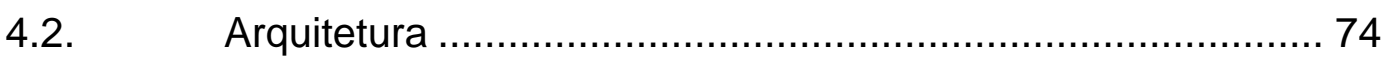

4.3. Equacionamento ........................................................ 76

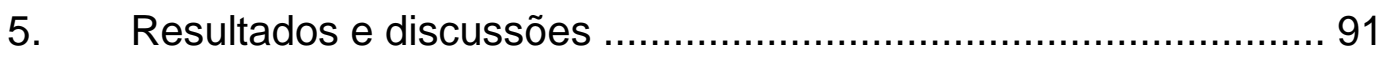

5.1. Levantamento de dados operacionais de Altos-Fornos de usinas siderúrgicas no Brasil e Exterior ................................. 91

5.2. Análise de sensibilidade do modelo para identificação dos parâmetros que mais impactam os resultados...................... 93

5.3. Validação do modelo ....................................................... 96

5.4. Estudo de caso ................................................................ 102

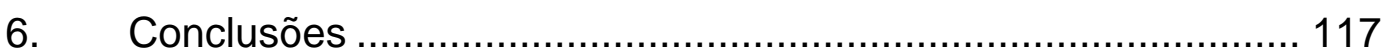

7. Sugestões para trabalhos futuros ............................................ 119

8. Referências bibliográficas ................................................... 120 


\section{Lista de Figuras}

Figura 1 - Evolução da produção de aço no mundo. ........................... 18

Figura 2 - Evolução da produção de aço no mundo.......................... 19

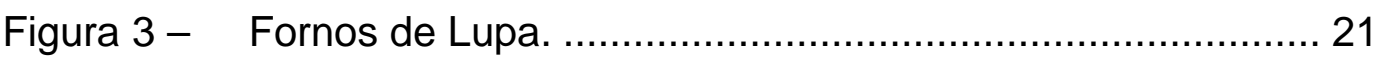

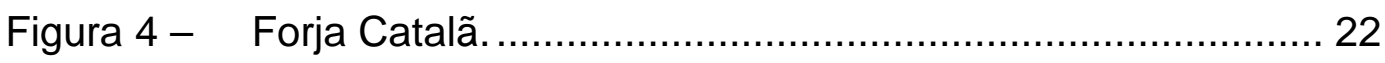

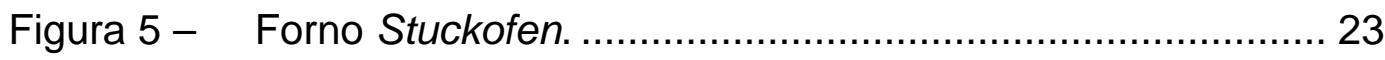

Figura 6 - Alto-Forno americano movido a rodas d'água..................... 24

Figura 7 - Corte esquemático do Alto-Forno...................................... 27

Figura 8 - Equipamentos auxiliares do Alto-Forno ............................. 28

Figura 9 - Sistema de carregamento de duplo cone.......................... 29

Figura 10 - Sistema de carregamento de calha rotativa. ...................... 30

Figura 11 - Coletor de Pó................................................................ 31

Figura 12 - Lavador de gases..................................................... 31

Figura 13 - Representação esquemática de um regenerador................ 32

Figura 14 - Estrutura interna de um Alto-Forno ................................... 35

Figura 15 - Esquema de divisão do Alto-Forno: modelo operacional e dissecação pelos japoneses............................................... 38

Figura 16 - Diagramas de equilíbrio Fe-C...................................... 41

Figura 17 - Diagrama de oxidação-redução dos sistemas Fe-C-H-O... 43

Figura 18 - Comparação entre o processo de redução.......................... 44

Figura 19 - Influência do tamanho médio de minérios granulados sobre a redutibilidade......................................................... 45

Figura 20 - Principais matérias-primas empregadas na produção de

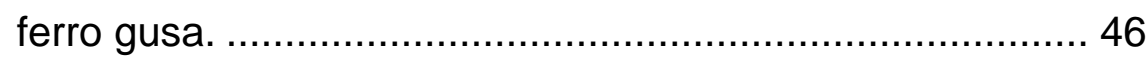

Figura 21- Exemplo de dois tipos de partículas que entram em

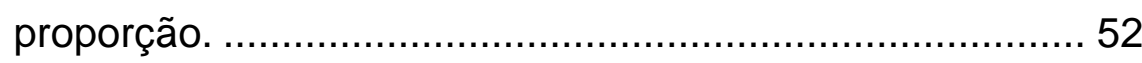

Figura 22 - Influência do diâmetro médio sobre o volume de vazios e a perda de carga através de um leito de pelotas................... 52

Figura 23 - Evolução dos preços de minério de ferro para o mercado

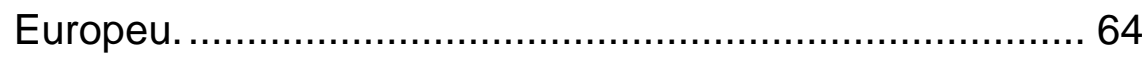

Figura 24 - Evolução do preço de sinter feed para distintos mercados. 68 
Figura 25 - Evolução do preço de pelotas para Alto-Forno para distintos mercados. 68

Figura 26 - Fluxo esquemático de pelotas e lump do porto do fornecedor até o destino final. 69

Figura 27 - Fluxo esquemático de sinter feed do porto do fornecedor até o destino final e a produção de sínter. 70

Figura 28 - Representação simplificada do processo de modelagem... 59

Figura 29 - Tela do modelo com os principais fluxos do processo de Alto-Forno. 75

Figura 30 - Tela do modelo para análise de Valor em Uso. 76

Figura 31 - Gráfico da relação entre coeficientes de atividade da cal e sulfeto de cálcio na escória e a relação à basicidade binária da escória do Alto-Forno 82

Figura 32 - Gráfico da relação do coeficiente $\delta \mathrm{SiO} 2$ e a basicidade binária da escória do Alto-Forno obedecendo à parametrização do teor de alumina. 85

Figura 33 - Gráfico da relação do coeficiente $\delta \mathrm{MnO}$ e a basicidade binária da escória do Alto-Forno obedecendo à parametrização do teor de alumina. 86

Figura 34 - Tela do modelo que ilustra a escória.................................. 88

Figura 35 - Tela do modelo que ilustra os fluxantes. ............................ 89

Figura 36 - Diagrama da arquitetura do modelo termoquímico............. 90

Figura 37 - Tela do banco de dados do modelo de Alto-Forno............. 92

Figura 38 - Tela do gráfico de sensibilidade do modelo de Alto-Forno. 93

Figura 39 - Análise química das cargas metálicas adotadas no estudo

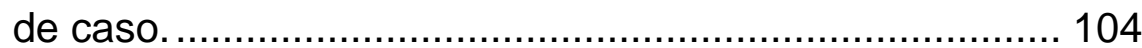

Figura 40 - Participação média das cargas metálicas nos Altos-Fornos do banco de dados. 105

Figura 41 - Distribuição média das cargas metálicas nos Altos-Fornos no

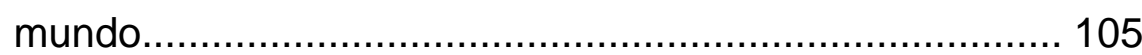

Figura 42 - Gráficos dos resultados dos cenários discutidos.............. 110

Figura 43 - Gráficos dos preços das cargas metálicas e do valor em uso da pelota 116 


\section{Lista de Tabelas}

Tabela 1 - Comparação do carvão vegetal com o coque. .................... 49

Tabela 2 - Alto-Forno a carvão vegetal x Alto-Forno a Coque.............. 49

Tabela 3 - Características a serem controladas das matérias-primas. 50

Tabela 4 - Características físicas, químicas e metalúrgicas típicas de minérios de ferro usados em Alto-Forno.............................. 57

Tabela 7 - Dados Termodinâmicos ...................................................... 80

Tabela 6 - Cálculo do impacto da umidade e teor de ferro no custo final do frete marítimo. ................................................................. 71

Tabela 8 - Relação dos Altos-Fornos utilizados no modelo.................. 91

Tabela 9 - Resultados da análise de sensibilidade dos parâmetros de entrada do modelo............................................................. 94

Tabela 10 - Resumo da análise de sensibilidade dos parâmetros de entrada do modelo............................................................ 95

Tabela 11 - Comparação dos resultados do modelo com os dados informados pelos operadores dos Altos-Fornos. ................ 98

Tabela 12 - Cenários utilizados para o estudo de caso....................... 106

Tabela 13 - Qualidade química do ferro gusa resultante dos cenários.106

Tabela 14 - Resultados dos cenários do estudo de caso. ................... 107 